\title{
Alterations of Caspr 2 and Nav1.6 on myelinated axon damage in a rat model of chronic cerebral hypoperfusion
}

\author{
WEIHUA LIANG ${ }^{1,2}$, WEIWEI ZHANG ${ }^{3}$, SHIFU ZHAO ${ }^{2}$, HUA LIANG $^{4}$, JINLI ZHANG $^{1}$ and LUYAN WANG $^{1}$ \\ ${ }^{1}$ No. 263 Clinic of PLA Army General Hospital, Beijing 101149; ${ }^{2}$ Department of Neurology, Xinqiao Hospital, \\ The Third Military Medical University, Chongqing 400038; ${ }^{3}$ PLA Army General Hospital, Beijing 100700; \\ ${ }^{4}$ The 66083rd of PLA, Beijing 102488, P.R. China
}

Received June 13, 2016; Accepted January 11, 2017

DOI: $10.3892 /$ etm.2017.4228

\begin{abstract}
Myelinated axons require the correct localization of key proteins that are essential for nerve conduction and cognitive function. Little is known regarding the altered expression of contactin-associated protein 2 (Caspr2) at the juxtaparanodal regions and Nav1.6 at the node of Ranvier in response to chronic cerebral hypoperfusion $(\mathrm{CCH})$. The aim of the present study was to examine the alterations in the key protein of myelinated axons and the potential mechanisms that may follow $\mathrm{CCH}$. We established a rat model of $\mathrm{CCH}$ by controllable partial narrowing of bilateral common carotid arteries. Then, we detected cerebral blood flow (CBF) after surgery. We also evaluated motor-evoked potentials (MEPs), assessed the Morris water maze test, analyzed Caspr2 expression through immunohistochemistry and Nav1.6 protein expression through western blot analysis at 2, 4 and 12 weeks. The results revealed that the mean $\mathrm{CBF}$ value was significantly decreased to $33.90 \pm 5.48 \%$. The MEP latencies and the escaping latencies were significantly prolonged. There was also an elongation of the first time passing of the hidden platform with a reduction of crossing platform times in spatial probing. Furthermore, the Caspr 2 immunoreactivity demonstrated that the Caspr 2 level was significantly downregulated with abnormal locations in the corpus callosum. The western blot analysis of Nav1.6 protein revealed that the level was reduced significantly over time. The results demonstrate that $\mathrm{CCH}$ leads to central conductive function loss, cognitive function damage and alterations in the key protein of myelinated axons, which may provide a molecular basis and key link for white matter damage.
\end{abstract}

Correspondence to: Dr Weiwei Zhang, PLA Army General Hospital, 5 Nan Men Cang, Beijing 100700, P.R. China

E-mail: weiwei_zhang111@163.com

Dr Shifu Zhao, Department of Neurology, Xinqiao Hospital, The Third Military Medical University, 183 Xinqiao Street, Chongqing 400038, P.R. China

E-mail: shifuzhao@163.com

Key words: chronic cerebral hypoperfusion, Caspr2, Nav1.6, white matter damage, myelinated axons

\section{Introduction}

Chronic cerebral hypoperfusion $(\mathrm{CCH})$ plays a key role in white matter (WM) damage, which contributes to the progression of dementias such as Alzheimer's disease and vascular dementia (1-5). The integrity of the brain's WM is comprised mainly of myelinated axons (6). Thus, the damage of myelinated axons associated with $\mathrm{CCH}$ results in development and progression of dementia. It is essential to determine early alterations of myelinated axons and potential mechanisms following $\mathrm{CCH}$, which provide an experimental basis for the prevention of dementia.

Myelination of axons consist of nodes of Ranvier, paranodal regions, juxtaparanodal regions, and internodes $(7,8)$. Each defined region contains the localization of key proteins that are constituents of either the axolemma or the myelin sheath $(9,10)$. Contactin-associated protein 2 (Caspr2) localizes to juxtaparanodal regions in association with a cell adhesion molecule of the neurexin superfamily and forms a complex with contactin 2 to generate a membrane scaffold that clusters Kv1 channels $(11,12)$. Voltage-gated sodium $\left(\mathrm{Na}^{+}\right)$channels are concentrated at nodes of Ranvier, which are separated from each other by myelin-covered internodes. Nodes of Ranvier are responsible for the generation and efficient propagation of axon potentials $(13,14)$. Currently, information on the altered expression of Caspr2 and Nav following $\mathrm{CCH}$ is limited.

In the present study, stenosis of the bilateral carotid common artery in adult Sprague-Dawley rats was used to study the nerve conduction, cognitive function and the altered expression of Caspr2 and Nav in order to reveal its underlying mechanism, and to further develop therapeutic treatments.

\section{Materials and methods}

Animals. Adult male Sprague-Dawley rats (6 months, $200 \pm 8.5 \mathrm{~g})$ were housed at a constant room temperature of $24^{\circ} \mathrm{C}$ on a 12 -h light-dark cycle, and maintained on an ad libitum diet (Experimental Animal Center, Third Military Medical University, China). All the experiments were performed with the approval of the Third Military Medical University Animal Ethics Committee. Every effort was made to minimize animal suffering and to keep the number of animals at a minimum. 
Bilateral common carotid artery stenosis (BCAS) surgery (surgical procedure). The rats were anaesthetized via intraperitoneal (i.p.) injection of $3 \%$ pentobarbital sodium (45 mg/kg) until their eyelash reflex disappeared, and were then fixed in the dorsal position. Under aseptic conditions, a $2-\mathrm{cm}$ incision was made in the median neck. After separation of the tissue layers, $\sim 1 \mathrm{~cm}$ of bilateral common carotid artery was separated from paratracheal carotid sheath, ligated using a 1.2-mm diameter needle together with no. 4 silk thread, and repositioned. The wound was closed, and the rats were given aspirin as anticoagulant $(30 \mathrm{mg} / \mathrm{l})$ in their drinking water for three days post-surgery. For rats in the sham group, the bilateral common carotid artery was exposed but no ligature was made. The animals were randomly allocated into four groups of six rats each: Sham, 2, 4 and 12 weeks.

Cerebral blood flow (CBF) measurement. The animals were anesthetized with sodium pentobarbital $(0.5 \mathrm{mg} / \mathrm{kg}$, i.p.), and the skin overlying the left skull was reflected. A plastic guide cannula ( $3 \mathrm{~mm}$ outer diameter, $2 \mathrm{~mm}$ inner diameter, $4 \mathrm{~mm}$ length) was fixed perpendicularly to the skull at $1 \mathrm{~mm}$ posterior and $2.5 \mathrm{~mm}$ lateral to bregma using dental resin. A 2.0-mm straight probe (Probe 418-1 Master probe) was inserted through the guide cannula, and $\mathrm{CBF}$ was recorded after the surgery using laser-Doppler flowmetry (PF5010LDPM; Perimed AB, Stockholm, Sweden). CBF values were expressed as a percentage of the baseline value.

Detection of the transcranial electrical stimulation motor-evoked potential (MEP). The transcranial electrical stimulation MEPs was detected ( $\mathrm{n}=6$ at each time point in every group) by Keypoint four guide evoked potential (Experimental Center, Third Military Medical University, China). All Sprague-Dawley rats were recorded at a quiet, electrically shielded room temperature of $24^{\circ} \mathrm{C}$. The rats were anaesthetized via i.p. injection of $3 \%$ pentobarbital sodium at a dose of $45 \mathrm{mg} / \mathrm{kg}$ until their eyelash reflex disappeared. EEG was detected with needle electrodes. The stimulation electrode was located at $1 \mathrm{~mm}$ posterior to the bregma. The recording electrode was located at $3 \mathrm{~mm}$ lateral to the sagittal suture. The recording electrode was located at the contralateral gastrocnemius. The monopolar recording on the nasal bone was ground. Electrical stimulation signals reveal a single square wave electrical pulse (stimulus intensity 5-12 mA, pulse width $0.2 \mathrm{msec}$ ). Each result was repeated at least twice to obtain a stable waveform. The distance between stimulating and recording electrodes was measured in all rats to derive an estimate of the MEP latency.

Morris water maze. Two, four, and 12 weeks after $\mathrm{CCH}$ surgery, each rat ( $n=6 /$ group) was subjected to a water maze test which consisted of a large circular pool (diameter, $2 \mathrm{~m}$; height, $1.5 \mathrm{~m})$ filled with water $\left(25 \pm 1^{\circ} \mathrm{C}\right)$ that was rendered opaque by the addition of enough milk to prevent the rats from seeing an underwater platform (5-cm diameter), located $3.5 \mathrm{~cm}$ below the surface. The day before the start of the training, the rats were acclimatized to water by being allowed to swim freely in a pool. To measure the acquisition learning behavior, navigation trials were performed daily (4-8 trials/day) for 5 consecutive days for each rat. The rats were trained to swim to a hidden platform that was situated below the surface of the water and was placed at the center of one of the four quadrants throughout training. During the four trials, each rat was randomly placed in one of the four starting positions. The rats were given $2 \mathrm{~min}$ to find the platform and to stay on it for $10 \mathrm{sec}$. If the animals failed to find the platform within the given time, they were gently guided to the platform and were allowed to stay on it for $10 \mathrm{sec}$. The rats were trained to find the hidden platform in the pool over 10 trials. To evaluate the rat's spatial retention ability, the space probe trails were carried out on day 6 . The platform was removed, and the total distance traveled while swimming and the swimming distance in the target quadrant for $1 \mathrm{~min}$ were recorded by the tracking system.

Histochemical evaluation of myelinated axon damage. Two, four, and twelve weeks after $\mathrm{CCH}$ surgery, each rat ( $n=6 /$ group) was anesthetized and perfused intracardially with phosphate-buffered saline, followed by $4 \%$ paraformaldehyde. The brains were post-fixed in ice-cold $4 \%$ paraformaldehyde for $2 \mathrm{~h}$, and then cryoprotected in a $30 \%$ sucrose solution. Coronal cryostat sections $(20-\mu \mathrm{m})$ were mounted onto poly-lysine coated slides. Similar brain slices were selected for immunohistochemistry labeling, respectively. For quantitative assessment of myelinated axons, damaged sections were immunostained using antibodies against Caspr2. Immunoreactivity was revealed by the primary antibody against Caspr2 (polyclonal rabbit anti-rat Caspr2 obtained from Boster Biological Technology Co. Ltd., Wuhan, China). FITC-labeled anti-rabbit secondary antibody was obtained from Beijing Zhongshan Golden Bridge Biotechnology Co., Ltd. (Beijing, China). A laser scanning confocal microscope was used to examine the presence and distribution of fluorescence in the sections. The Image-Pro Plus analysis system was used to determine the average optical density (AOD) value of expression.

Examination of Nav protein levels by western blot analysis. For total protein extraction, tissues were transferred to cold lysis buffer containing $0.1 \mathrm{~mol} / 1 \mathrm{NaCl}, 0.05 \mathrm{~mol} / 1$ Tris- $\mathrm{HCl}$ (pH 7.6), 0.001 mol/1 EDTA (pH 8.0), 0.1\% Tween-20, aprotinin $(1 \mu \mathrm{g} / \mathrm{ml})$, and PMSF $(100 \mu \mathrm{g} / \mathrm{ml})$ and homogenized on ice. The homogenate was centrifuged at $8,500 \mathrm{x} g$ for $10 \mathrm{~min}$, and the supernatant was stored at $4^{\circ} \mathrm{C}$. Equal amounts of protein from each sample $(50 \mu \mathrm{g})$ were mixed with $30-35 \mu \mathrm{l}$ sample buffer and boiled for $4 \mathrm{~min}$. Samples were separated by electrophoresis on a $10 \%$ SDS-PAGE gel and transferred to nitrocellulose membranes at $100 \mathrm{~mA}$ for $2 \mathrm{~h}$. The membrane was blocked with 5\% non-fat dry milk in PBS containing $0.01 \%$ Tween- 20 at $4^{\circ} \mathrm{C}$ overnight. Immunoblots were probed with specific antibodies: Polyclonal rabbit anti-rat Caspr2 (1:1,000; cat. no. TA326393; Beijing Zhongshan Golden Bridge Biotechnology Co., Ltd.). Blots were washed and incubated with horseradish peroxidase-conjugated anti-rabbit antibodies (1:2,000; cat. no. ZDR-5306; Beijing Zhongshan Golden Bridge Biotechnology Co., Ltd.) for $1 \mathrm{~h}$ at $25^{\circ} \mathrm{C}$. An endogenous control protein, GAPDH, was included in each western blot analysis. Then the membranes were developed using enhanced chemiluminescence reagent. The optical densities of the specific bands were scanned and measured by 


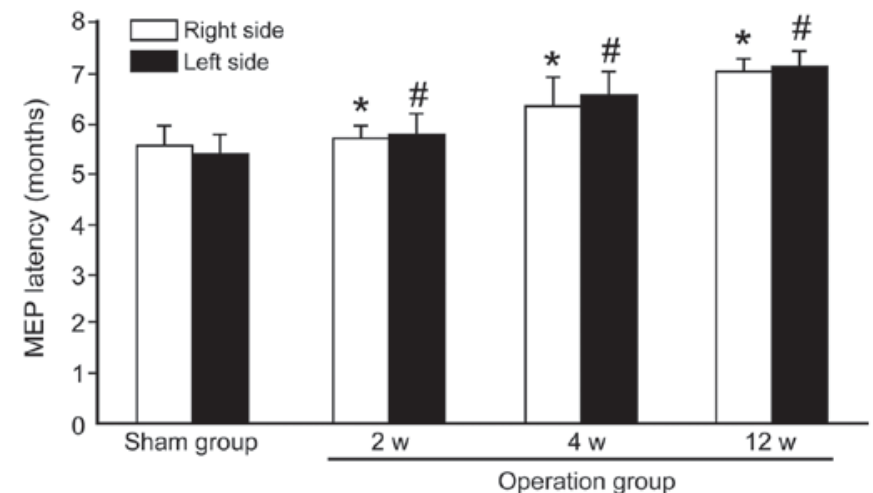

Figure 1. The latency of motor-evoked potential (MEP) was recorded at 2, 4 and 12 weeks after surgery. $n=6,{ }^{*} \mathrm{P}<0.05$ compared with the right side of rat in the sham group, ${ }^{\mathrm{P}} \mathrm{P}<0.05$ compared with the left side of rat in the sham group.

image analysis software (Labworks 4.6, China) and normalized to GAPDH.

Statistical analysis. Statistical analysis was performed using the SPSS 13.0 software package (Chicago, IL, USA). Values were expressed as means \pm standard deviation (SD). Analysis of variance was used to determine the significance of differences for multiple comparisons. $\mathrm{P}<0.05$ was considered to indicate a statistically significant difference.

\section{Results}

Changes of $C B F$ in the rats after surgery. $\mathrm{CBF}$ values were recorded for all the animals prior to and after surgery using laser-Doppler flowmetry. Compared to the sham group, CBF values in the operation group decreased significantly after surgery. The mean $\mathrm{CBF}$ value in rats undergoing $\mathrm{CCH}$ surgery was reduced significantly to $33.90 \pm 5.48 \%$ compared to that of sham animals $(\mathrm{P}<0.05)$. There were no marked differences in $\mathrm{CBF}$ in the three stenosis groups.

Transcranial electrical stimulation MEP. After 2, 4 and 12 weeks of surgery, the MEP latency in the operated groups was significantly prolonged compared to the values prior to the sham group (Fig. 1). Compared with the sham group (right $5.50 \pm 0.41 \mathrm{vs}$. left $5.38 \pm 0.37 \mathrm{msec}$ ), the MEP latencies of the operated groups were significantly longer (right $5.67 \pm 0.23$ vs. left $5.75 \pm 0.27 \mathrm{msec}$ at 2 weeks; right $6.25 \pm 0.58$ vs. left $6.50 \pm 0.44 \mathrm{msec}$ at 4 weeks and right $7.05 \pm 0.16 \mathrm{vs}$. left $7.08 \pm 0.29 \mathrm{msec}$ at 12 weeks; $\mathrm{P}<0.05)$. The MEP latencies had no significant change between the left and the right sides of the rat either in the sham group or the operated groups $(\mathrm{P}>0.05)$.

Morris water maze. Morris water maze analyses show that cognitive functions were markedly damaged at 2, 4 and 12 weeks in the operated groups following $\mathrm{CCH}$. Compared to the sham group, the escaping latency of the operation group was significantly prolonged (Fig. 2). In addition, times of crossing platform $(2.8 \pm 0.35$ at 2 weeks; $2.1 \pm 0.38$ at 4 weeks; $1.2 \pm 0.50$ at 12 weeks) in spatial probing were markedly decreased in the operation groups as compared with the sham group $(3.2 \pm 0.77)(\mathrm{P}<0.05)$. Furthermore, the first time

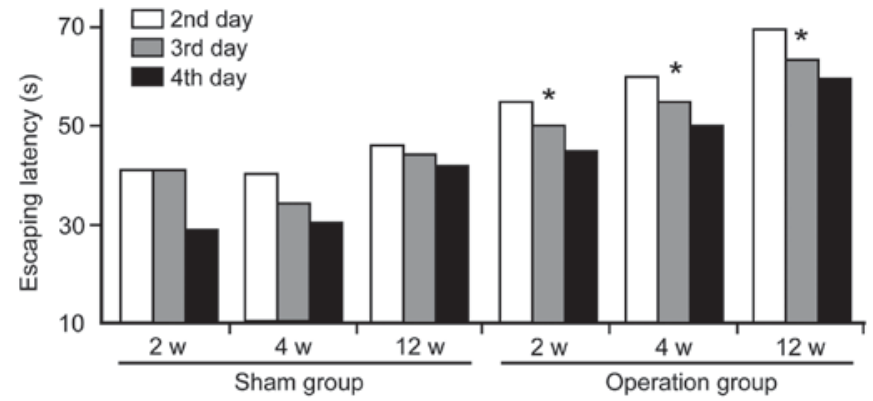

Figure 2. The escaping latency was recorded at 2, 4 and 12 weeks after surgery. There was a significant difference between the sham and operation groups. $\mathrm{n}=6$ in each group, ${ }^{*} \mathrm{P}<0.05$ compared with the sham group.

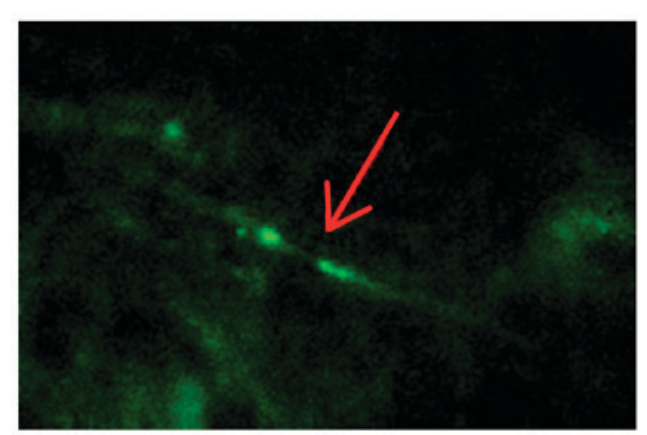

Figure 3. Amplified image of contactin-associated protein 2 (Caspr2)-positive particle at a Ranvier node, showing a model demonstrating the structure of the Ranvier node.

passing hidden platform $(49 \pm 3.16 \mathrm{sec}$ at 2 weeks; $65 \pm 2.54 \mathrm{sec}$ at 4 weeks; $91.5 \pm 3.80 \mathrm{sec}$ at 12 weeks) was prolonged significantly in the operation groups when compared to the sham group $(34 \pm 2.94 \mathrm{sec})(\mathrm{P}<0.05)$.

Histochemical evaluation of myelinated axon damage. Histological analyses revealed that the myelinated axons were significantly damaged at 2, 4 and 12 weeks in the operation groups. Compared to the sham group, $\mathrm{CCH}$ leads to a large change in Caspr 2 protein expression. Caspr 2 protein expression was detected as fine green particles using immunofluorescence (Fig. 3). As shown in Fig. 4, both the number and intensity of the particles decreased significantly. Moreover, the intensity of stain was reduced markedly over time. The distribution pattern of Caspr2 expression also changed markedly. Compared to the sham group, our results showed the Caspr2 expression was disordered in the distribution of strip and point in the corpus callosum following $\mathrm{CCH}$. At 12 weeks after the model preparation, the Caspr2-positive particles gathered around the area.

Examination of Nav1.6 protein levels by western blot analysis. Protein levels of Nav1.6 of each group were determined by western blot analysis. Compared to the sham group, Nav1.6 protein levels decreased markedly following $\mathrm{CCH}$ (Fig. 5A). In addition, the level of Nav1.6 was reduced over time. As shown in Fig. 5B, the Nav1.6 expression following $\mathrm{CCH}$ was significantly downregulated in the operation groups when compared to the sham group $(\mathrm{P}<0.05)$. Furthermore, there was a marked difference between the groups at different phases. 

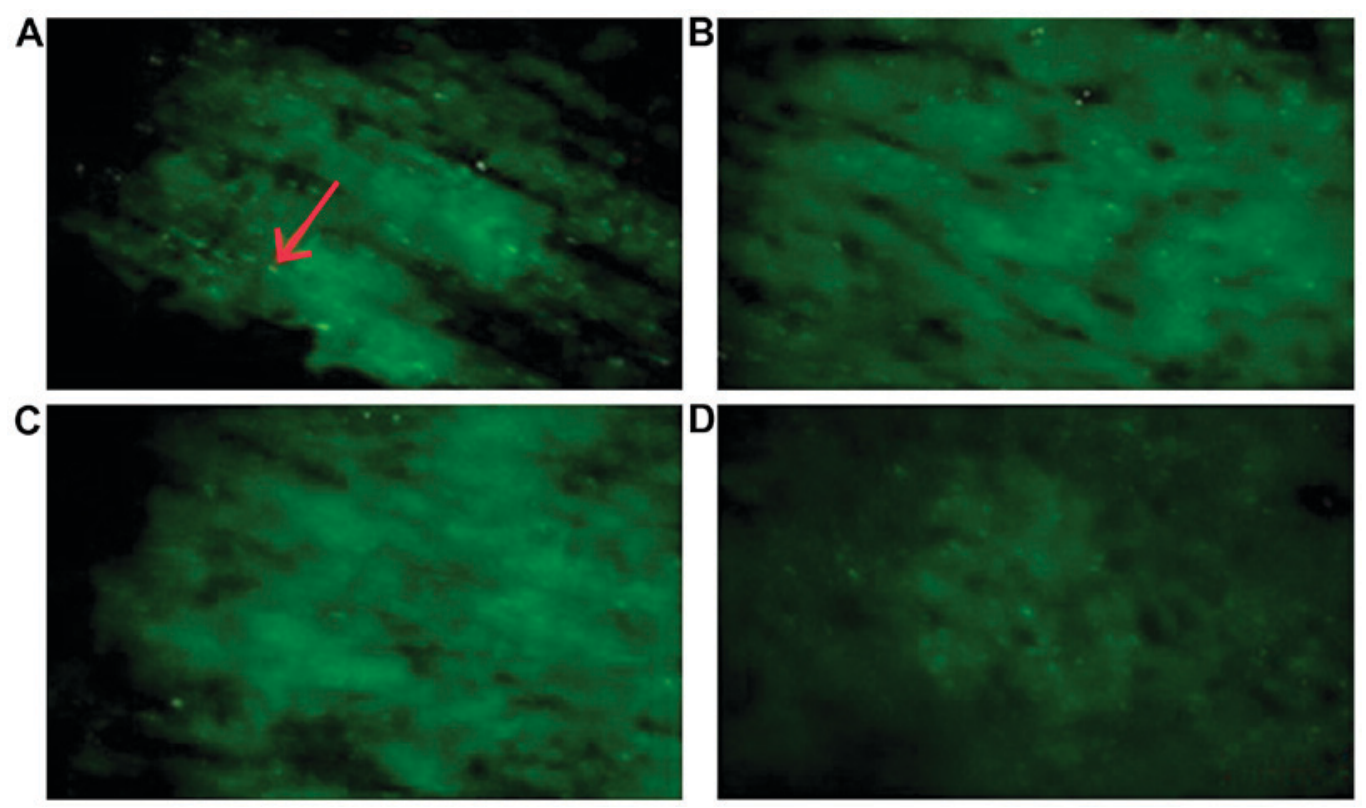

Figure 4. Immunoreactivity of contactin-associated protein 2 (Caspr2) in the corpus callosum at 2, 4 and 12 weeks after surgery. (A) Sham group, arrows indicate the expression of caspr2 protein; (B) at 2 weeks in operation group; (C) at 4 weeks in operation group; (D) at 12 weeks in operation group (scale bar, $20 \mu \mathrm{m}$ ).

A

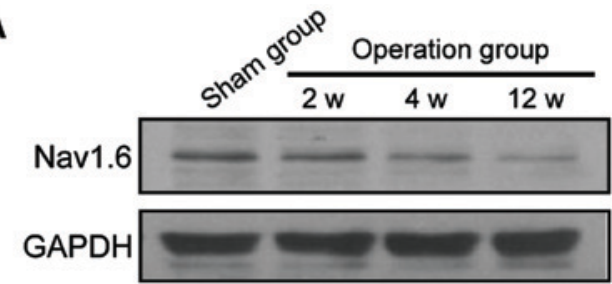

B

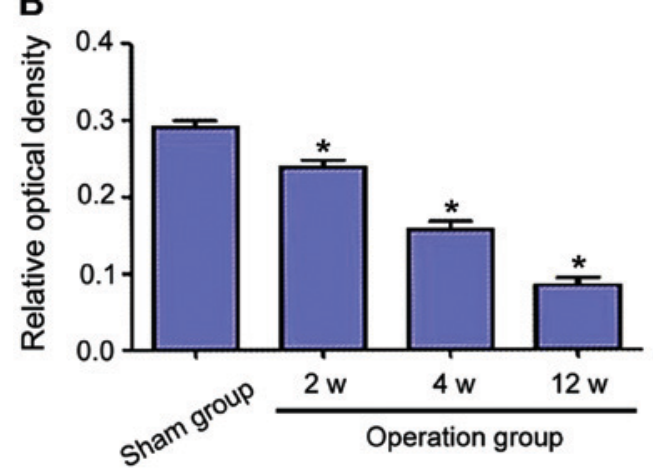

Figure 5. Nav1.6 protein expresion at 2, 4 and 12 weeks. (A) Nav1.6 protein detected by western blot analysis of at 2,4 and 12 weeks after surgery. (B) The relative optical density value of Nav1.6 protein at 2, 4 and 12 weeks after surgery. $n=6,{ }^{*} \mathrm{P}<0.05$ compared with the sham group.

\section{Discussion}

The present results have shown a progression of the damage to myelinated axons over time following $\mathrm{CCH}$. In order to understand the mechanisms and quantify the myelinated axon damage, we observed the loss of nerve conduction, the damage of cognitive function and the alterations of Caspr2 and Nav1.6 levels in a rat model of $\mathrm{CCH}$. The data have demonstrated that Caspr2 levels were significantly downregulated with abnormal localization. At the same time, Nav1.6 levels were reduced markedly following $\mathrm{CCH}$.
This study has demonstrated that the nerve conduction and cognitive function reduced markedly after $\mathrm{CCH}$ in animals, which is consistent with a chronic persistent decrease of CBF. These results indicate that very early determination of cognitive function and MEP in patients can provide a promising strategy for judgment of $\mathrm{CCH}$ and prevention of dementia in clinic.

Recent studies conducted on the correlation of Caspr2 and Nav expression to damage of myelinated axons have focused on the potential mechanisms of demyelination disease (15-17). Some studies indicate that the Caspr2 and Nav expression may be involved in a progressive axonal denudation and nerve conduction impairment, which contributes to the demyelination pathology (15-17). However, studies related to the influence of Caspr2 and Nav expression on WM damage induced by $\mathrm{CCH}$ are limited. In the present study, alterations in the key proteins of myelinated axons were observed in a rat model of $\mathrm{CCH}$. The immunohistochemical analysis of Caspr2 protein revealed that the level and localization changed significantly. Compared to the sham group, the number and intensity of the particles decreased significantly over time. Caspr2 expression was found to be disordered in the locations of strip and point in the corpus callosum following $\mathrm{CCH}$. At 12 weeks after the operation, the Caspr 2 particles gathered around in part of the area. Thus, the decreasing expression and abnormal locations of the Caspr 2 protein contributed to the myelinated axon damage induced by $\mathrm{CCH}$. Consistent with the above results, previous studies showed that Caspr2 expression was downregulated with disruption of normal localization and diffusion in multiple sclerosis $(18,19)$. Therefore, $\mathrm{CCH}$ results in the alterations of Caspr 2 expression, which contributes to myelinated axon damage. In addition, the western blot analysis of Nav1.6 protein revealed that the level of this protein reduced significantly over time, which provided further support that $\mathrm{CCH}$ causes damage to myelinated axons. Similarly, previous studies showed that the alteration in the 
expression of Nav1.6 contributed to the damage to myelinated axons, which occurred in a variety of neuropathological states including traumatic brain injury $(20,21)$, spinal injury $(22,23)$, demyelination disease $(24,25)$ and epilepsy $(26,27)$. Moreover, it was observed that there was a time-dependent loss of Nav mRNA and protein in response to $\mathrm{CCH}$ as a result of focal cerebral ischemia $(28,29)$, which corroborates the results of the present study.

Taken together, this study revealed that there is progression of Caspr2 and Nav1.6 expression in rat model of $\mathrm{CCH}$ over time. We suggested that the alterations of Caspr2 and Nav1.6 on myelinated axon damage after $\mathrm{CCH}$ may be in response to the loss of nerve conduction and the damage of cognitive function, which provided molecular basis for the prevention of dementia. However, the present study revealed that the molecular mechanisms induced by $\mathrm{CCH}$ were limited. There is emerging evidence on distinct protein architecture alterations after mild cerebral hypoperfusion (30). Thus, the complexity of the molecular mechanisms induced by $\mathrm{CCH}$ suggests that additional experiments are required to clarify the WM damage.

In conclusion, our results demonstrate that $\mathrm{CCH}$ results in time-dependent loss of Caspr2 and Nav1.6 protein, which contributes to the damage of myelinated axons. These results provide insight into the mechanisms for WM damage in response to $\mathrm{CCH}$.

\section{References}

1. Akinyemi RO, Mukaetova-Ladinska EB, Attems J, Ihara M and Kalaria RN: Vascular risk factors and neurodegeneration in ageing related dementias: Alzheimer's disease and vascular dementia. Curr Alzheimer Res 10: 642-653, 2013.

2. Hainsworth AH, Brittain JF and Khatun H: Pre-clinical models of human cerebral small vessel disease: utility for clinical application. J Neurol Sci 322: 237-240, 2012.

3. Miki K, Ishibashi S, Sun L, Xu H, Ohashi W, Kuroiwa T and Mizusawa H: Intensity of chronic cerebral hypoperfusion determines white/gray matter injury and cognitive/motor dysfunction in mice. J Neurosci Res 87: 1270-1281, 2009.

4. Brown WR, Moody DM, Thore CR, Anstrom JA and Challa VR: Microvascular changes in the white mater in dementia. J Neurol Sci 283: 28-31, 2009.

5. Fernando MS, Simpson JE, Matthews F, Brayne C, Lewis CE, Barber R, Kalaria RN, Forster G, Esteves F, Wharton SB, et al; MRC Cognitive Function and Ageing Neuropathology Study Group: White matter lesions in an unselected cohort of the elderly: molecular pathology suggests origin from chronic hypoperfusion injury. Stroke 37: 1391-1398, 2006.

6. Nave K-A: Myelination and support of axonal integrity by glia. Nature 468: 244-252, 2010.

7. Salzer JL: Clustering sodium channels at the node of Ranvier: close encounters of the axon-glia kind. Neuron 18: 843-846, 1997.

8. Arroyo EJ and Scherer SS: On the molecular architecture of myelinated fibers. Histochem Cell Biol 113: 1-18, 2000.

9. Rios JC, Rubin M, St Martin M, Downey RT, Einheber S, Rosenbluth J, Levinson SR, Bhat M and Salzer JL: Paranodal interactions regulate expression of sodium channel subtypes and provide a diffusion barrier for the node of Ranvier. J Neurosci 23: 7001-7011, 2003

10. Susuki K and Rasband MN: Molecular mechanisms of node of Ranvier formation. Curr Opin Cell Biol 20: 616-623, 2008.

11. Peles E and Salzer JL: Molecular domains of myelinated axons. Curr Opin Neurobiol 10: 558-565, 2000.

12. Girault JA, Oguievetskaia K, Carnaud M, Denisenko-Nehrbass N and Goutebroze L: Transmembrane scaffolding proteins in the formation and stability of nodes of Ranvier. Biol Cell 95: 447-452, 2003.
13. Gasser A, Ho TS-Y, Cheng X, Chang K-J, Waxman SG, Rasband MN and Dib-Hajj SD: An ankyrinG-binding motif is necessary and sufficient for targeting Nav1.6 sodium channels to axon initial segments and nodes of Ranvier. J Neurosci 32: 7232-7243, 2012.

14. Leterrier C, Brachet A, Dargent B and Vacher H: Determinants of voltage-gated sodium channel clustering in neurons. Semin Cell Dev Biol 22: 171-177, 2011.

15. Coman I, Aigrot MS, Seilhean D, Reynolds R, Girault JA, Zalc B and Lubetzki C: Nodal, paranodal and juxtaparanodal axonal proteins during demyelination and remyelination in multiple sclerosis. Brain 129: 3186-3195, 2006.

16. Howell OW, Palser A, Polito A, Melrose S, Zonta B Scheiermann C, Vora AJ, Brophy PJ and Reynolds R: Disruption of neurofascin localization reveals early changes preceding demyelination and remyelination in multiple sclerosis. Brain 129: 3173-3185, 2006.

17. Howell OW, Rundle JL, Garg A, Komada M, Brophy PJ and Reynolds R: Activated microglia mediate axoglial disruption that contributes to axonal injury in multiple sclerosis. J Neuropathol Exp Neurol 69: 1017-1033, 2010.

18. Wolswijk G and Balesar R: Changes in the expression and localization of the paranodal protein Caspr on axons in chronic multiple sclerosis. Brain 126: 1638-1649, 2003.

19. Zoupi L, Markoullis K, Kleopa KA and Karagogeos D: Alterations of juxtaparanodal domains in two rodent models of CNS demyelination. Glia 61: 1236-1249, 2013.

20. Mao Q, Jia F, Zhang XH, Qiu YM, Ge JW, Bao WJ, Luo QZ and Jiang JY: The up-regulation of voltage-gated sodium channel Nav1.6 expression following fluid percussion traumatic brain injury in rats. Neurosurgery 66: 1134-1139, discussion $1139,2010$.

21. Wang JALW, Lin W, Morris T, Banderali U, Juranka PF and Morris CE: Membrane trauma and $\mathrm{Na}^{+}$leak from Nav1.6 channels. Am J Physiol Cell Physiol 297: C823-C834, 2009.

22. Hunanyan AS, Alessi V, Patel S, Pearse DD, Matthews G and Arvanian VL: Alterations of action potentials and the localization of Nav1.6 sodium channels in spared axons after hemisection injury of the spinal cord in adult rats. J Neurophysiol 105: 1033-1044, 2011 .

23. Wienecke J, Westerdahl A-C, Hultborn H, Kiehn O and Ryge J: Global gene expression analysis of rodent motor neurons following spinal cord injury associates molecular mechanisms with development of postinjury spasticity. J Neurophysiol 103: 761-778, 2010.

24. Hassen GWFJ, Feliberti J, Kesner L, Stracher A and Mokhtarian F: Prevention of axonal injury using calpain inhibitor in chronic progressive experimental autoimmune encephalomyelitis. Brain Res 1236: 206-215, 2008.

25. Black JA, Newcombe J, Trapp BD and Waxman SG: Sodium channel expression within chronic multiple sclerosis plaques. J Neuropathol Exp Neurol 66: 828-837, 2007.

26. Liao Y, Deprez L, Maljevic S, Pitsch J, Claes L, Hristova D, Jordanova A, Ala-Mello S, Bellan-Koch A, Blazevic D, et al: Molecular correlates of age-dependent seizures in an inherited neonatal-infantile epilepsy. Brain 133: 1403-1414, 2010.

27. Yao C, Williams AJ, Cui P, Berti R, Hunter JC, Tortella FC and Dave JR: Differential pattern of expression of voltage-gated sodium channel genes following ischemic brain injury in rats. Neurotox Res 4: 67-75, 2002.

28. Blumenfeld H, Lampert A, Klein JP, Mission J, Chen MC, Rivera M, Dib-Hajj S, Brennan AR, Hains BC and Waxman SG: Role of hippocampal sodium channel Nav1.6 in kindling epileptogenesis. Epilepsia 50: 44-55, 2009.

29. Yao C, Williams AJ, Hartings JA, Lu XC, Tortella FC and Dave JR: Down-regulation of the sodium channel $\mathrm{Na}(\mathrm{v}) 1.1$ $\alpha$-subunit following focal ischemic brain injury in rats: in situ hybridization and immunohistochemical analysis. Life Sci 77: 1116-1129, 2005.

30. Reimer MM, McQueen J, Searcy L, Scullion G, Zonta B, Desmazieres A, Holland PR, Smith J, Gliddon C, Wood ER, et al: Rapid disruption of axon-glial integrity in response to mild cerebral hypoperfusion. J Neurosci 31: 18185-18194, 2011. 